\title{
LEVEL OF NEUTROPHIL GELATINASE-ASSOCIATED LIPOCALIN (NGAL) URIN AS A PREDICTOR OF ACUTE KIDNEY INJURY IN SEPSIS PATIENTS TREATED IN ICU H.ADAM MALIK MEDAN HOSPITAL
}

\section{Yudhistira \\ Burhanuddin \\ Nasution* \\ Achsanuddin \\ Hanafie}

Nindia Sugih Arto
Department Of Clinical Pathology, Faculty Of Medicine, Universitas Sumatera Utara / H. Adam Malik Hospital Medan

Department Of Clinical Pathology, Faculty Of Medicine, Universitas Sumatera Utara / H. Adam Malik Hospital Medan *Corresponding Author

Department Of Anesthesiology And Intensive Therapy, Faculty Of Medicine, Universitas Sumatera Utara / H. Adam Malik Hospital Medan

Department Of Clinical Pathology, Faculty Of Medicine, Universitas Sumatera Utara / H. Adam Malik Hospital Medan

ABSTRACT BACKGROUND: Sepsis is a complex interaction between the direct toxic effects of infecting microor ganisms and the disruption of the host's normal inflammatory response to infection. One organ that is often disrupted is the kidney . Neutrophil Gelatinase-Associated Lipocalin (NGAL) is an AKI biomarker that can be used as an early biomarker for AKI prediction, monitoring clinical trials in AKI and for the prognosis of AKI.

METHOD: This research is an observational study. 30 subjects with sepsis were examined for PCT, BUN, urea, creatinine and urine NGAL tests.

RESULTS: Statistical analysis using the Spearman Rank Test shows that NGAL level 1 has a significant relationship with PCT day $l$, with a value $(r=0.912)$ and $a$ value of $p<0.05$. NGAL level day $l$ did not have a significant relationship with levels of urea, creatinine, BUN with values $(r=0.113),(r=0.329),(r=0.352)$ and values $(p>0.05)$. NGAL day 3 had $a$ significant relationship with PCT day 1 , with a value $(r=0.0771)$ and a p value $<0.001$. Measurement of NGAL day 3 had a significant relationship with urea, creatinine, BUN, and values $(r=0.427),(r=0.550),(r=0.411)$ with a p value $<0.05$.

CONCLUSIONS AND RECOMMENDATIONS: From the results of the study, it was concluded that there was a difference between urine NGAL levels in septic patients on day 1 (197.26 \pm 118.54$)$ and day $3(288.31 \pm 136.96)$. There was a significant correlation between urinary NGAL levels with urea levels $(p=0.024 \& r=0.427)$ and creatinine $(p=0.002 \& r=0.550)$ on day 3 , so urine NGAL was a predictor of AKI events in sepsis patients on day l -3..

\section{KEYWORDS : Sepsis, Aki, Ngal}

\section{INTRODUCTION}

Sepsis is still the leading cause of death in critical cases throughout the world. The high incidence and problem of infection which is usually associated with developing countries or places with less hygiene, is apparently not entirely true. Data from the Center for Disease Control (CDC) shows that the incidence of sepsis increased by $\pm 8.7 \%$ every year, from 164,000 cases ( 83 per 100,000 population) in 1979 to 660,000 cases ( 240 cases per 100,000 population) in 2000 .

Sepsis is a life-threatening state of organ dysfunction where there is a dysregulation of the body's response to infection. Clinically it can be explained that organ dysfunction has an increased score on sequential organ failure assessment (SOFA). Sepsis is usually caused by a bacterial infection (although sepsis can be caused by a virus, or more often, is caused by a fungus). Causal microorganisms that are most commonly found in adults are Escherichia coli, Staphylococcus aureus, and Streptococcus pneumonia. Species Enterococcus, Klebsiella sp, and Pseudomonas are also often found. ${ }^{2}$

Acute kidney injury (AKI) becomes a complication in acute care admission patients and reaches $30 \%$ in patients admitted in intensive care units (ICU). Severe sepsis is the trigger for $50 \%$ of cases of AKI in critical patients. Impaired kidney function due to sepsis is characterized by decreased urine production, increased blood urea nitrogen (BUN), and creatinine. Severe sepsis is a trigger for $50 \%$ of cases of AKI in critical patients.

Neutrophil Gelatinase-Associated Lipocalin (NGAL) is a 25
$\mathrm{kD}$ molecular weight protein bound to gelatinase from neutrophil cells. In normal circumstances NGAL is excreted in very low levels from various body tissues, such as kidneys, lungs, stomach and colon. ${ }^{4}$ NGAL protein expression is detected in injured kidneys in tubular epithelial cells. ${ }^{4}$ NGAL can be examined from blood or urine and can be used to establish an early diagnosis of AKI because it can be detected more quickly before the rise in serum creatinine (about 1 to 2 days $)^{5}$

\section{METHOD}

The study was conducted at the Department of Clinical Pathology, Faculty of Medicine, Universitas Sumatera Utara / RSUP Haji Adam Malik Medan in collaboration with the Department of Anesthesiology and Intensive Therapy at the Faculty of Medicine, Universitas Sumatera Utara. This study was an observational study with a cohort study design. The study was conducted in April until July 2019. The research subjects were male and female patients treated at the ICU General Hospital. H. Adam Malik Medan who was diagnosed with sepsis.

The sample size in this study was determined at 30 samples. The inclusion criteria in this study were patients who met the criteria for sepsis who were treated at ICU H. Adam Malik General Hospital Medan, aged $>18$ years and $<65$ years, and agreed to participate in the study. The exclusion criteria are chronic kidney failure, the patient's autoimmune disease and malignancy. Each urine NGAL examination was performed on days 1 and 3. Urine NGAL examination was examined using an Architect with the principle of Chemiluminescent microparticle immunoassay (CMIA) examination. 
III. STATISTICS ANALYSIS

The results of the study were analyzed statistically using SPSS (Statistical Package for Social Sciences, Chicago, IL, USA) software for Windows. The description of the characteristics of the research subjects is presented in tabulated form and described. The relationship between urine NGAL days 1 and 3 is associated with levels of BUN, urea, creatinine and procalcitonin days 1 and 3 using the Spearman Correlation test.

\section{RESULT}

The study was conducted at the Department of Clinical Pathology, Faculty of Medicine, Universitas Sumatera Utara / H. Adam Malik Hospital Medan in collaboration with the Department of Anesthesiology and Intensive Therapy, Faculty of Medicine, Universitas Sumatera Utara / H. Adam Malik Hospital Medan. The study was conducted for 3 months from April to July 2019, of 30 patients who had gone through the process of inclusion and exclusion. The number of patients in the study sample were 30 patients ( 19 male and 11 female). 5 patients were excluded from the study because they died less than 48 hours in ICU care (Table 1)

Table.3 Descriptive Analysis of Āge, PCT, Ureum, Creatinine, BUN day 1, BUN day 3, and NGAL levels

\begin{tabular}{|c|c|c|c|c|}
\hline & \multicolumn{2}{|c|}{ Hari l } & \multicolumn{2}{c|}{ Hari 3 } \\
\hline Variabel & Mean \pm sd & Median (Min-Max) & Mean \pm sd & Median (Min-Max) \\
\hline Ureum & $30,27 \pm 9,12$ & $30,5(15-51)$ & $48,00 \pm 6,15$ & $48,00(35-58)$ \\
\hline Creatinin & $0,86 \pm 0,22$ & $0,85(0,46-1,25)$ & $2,18 \pm 0,31$ & $2,11(1,75-2,83)$ \\
\hline BUN & $18,10 \pm 4,06$ & $18,5(10-25)$ & $24,37 \pm 4,54$ & $24(17-34)$ \\
\hline NGAL & $197,26 \pm 118,54$ & $166,15(28,5-561,2)$ & $288,31 \pm 136,96$ & $255,95(111,8-592,2)$ \\
\hline
\end{tabular}

*) Descriptive analysis shows the Median because the data variables are not normally distributed.

Descriptive analysis shows the Median because the data variables are not normally distributed. On the measurement of ureum day 1 the median value is 30.5 (15-51), while the measurement of ureum day 3 is obtained a median value of 48.00 (35-58). Measuring creatinine day 1 obtained a median value of 0.85 (0.46-1.25), while creatinine measurement day 3 obtained a median value of 2.11 (1.75-2.83). Likewise, measurement of BUN on day 1 obtained a median value of 18.5 (10-25), whereas measurement of BUN on day 3 obtained a median value of 24 (17-34). Measurement of NGAL levels on day 1 obtained a median value of 166.15 (28.5-561.2), while measurement of NGAL levels on day 3 obtained a median value of 255.95 (1 1 1.8-592.2). (Table.3)

Analysis of Relationship of NGAL Levels with PCT, Ureum, Creatinine and BUN Days 1 and 3.

Table.4 Spearman NGAL Correlation Test with PCT, Ureum, Creatinine, BUN Day 1

\begin{tabular}{|l|c|c|c|}
\hline \multirow{2}{*}{ Variabel } & \multicolumn{3}{|c|}{ Kadar NGAL } \\
\cline { 2 - 4 } & $\mathbf{N}$ & $\mathbf{r}$ & $\mathbf{P}$ \\
\hline PCT & 30 & 0,912 & 0,0001 \\
\hline \multirow{2}{*}{ Ureum } & & 0,113 & 0,551 \\
\hline Creatinin & & 0,329 & 0,075 \\
\cline { 3 - 4 } BUN & & 0,352 & 0,057 \\
\hline
\end{tabular}

*) Correlation analysis uses the Spearman correlation test because the data are not normally distributed

In statistical analysis using the Spearman Rank Test correlation test because the data is not normally distributed. Spearman correlation test statistic test showed NGAL level 1 day had a significant relationship with PCT level 1 day, with a value $(r=0.912)$ and $a$ value of $p<0.05$. Whereas NGAL levels on day 1 did not have a significant relationship with levels of ureum, creatinine and BUN with each value $(r=0.113)$, $(r=$ $0.329)$, $(r=0.352)$ and each value $(p>0.05)$
Table.1 Descriptive Data Analysis of Gender in Sepsis Pati ents

\begin{tabular}{|l|c|c|}
\hline \multicolumn{1}{|c|}{ SEX } & N & $\%$ \\
\hline Male & 19 & $63,3 \%$ \\
\hline Female & 11 & $36,7 \%$ \\
\hline Total & $\mathbf{3 0}$ & $100 \%$ \\
\hline
\end{tabular}

*) Variable-scale variable categorical.

From statistical analysis of data on age and Procalcitonin (PCT) levels were not normally distributed, then the median value was used. Of all study participants, they had a median age of 50 years. The youngest age is 23 years and the oldest is 64 years. PCT levels with a median value of 50.5 , with the lowest level 0 and the highest level 98.34. (Table 2)

Table.2 Descriptive Data Analysis of Age and PCT Levels

\begin{tabular}{|l|l|l|l|}
\hline VARIABEL & N & Median & Min - Max \\
\hline Age & 30 & 50,0 & $23-64$ \\
\hline PCT & 30 & 50,5 & $0,00-98,34$ \\
\hline
\end{tabular}

*) Age variables and PCT content were not normally distri buted

While the measurement of NGAL day 3 has a significant relationship with PCT level day 1 , with a value $(r=0.0771)$ and $\alpha$ value of $p<0.001$. Likewise, measurement of NGAL day 3 has a significant relationship with levels of ureum, creatinine and BUN, with each value $(r=0.427),(r=0.550),(r=0.411)$ with each value of $p<0,05$. (Table.5)

Table.5 Spearman NGAL Correlation Test with PCT, Ureum, Creatinine, BUN Day 3

\begin{tabular}{|l|c|c|c|}
\hline \multirow{2}{*}{ Variabel } & \multicolumn{3}{|c|}{ NGAL LEVEL } \\
\cline { 2 - 4 } & $\mathbf{N}$ & $\mathbf{R}$ & $\mathbf{P}$ \\
\hline PCT & 30 & 0,771 & 0,0001 \\
\hline Ureum & & 0,427 & 0,019 \\
\cline { 1 - 1 } Creatinin & & 0,550 & 0,002 \\
\hline BUN & & 0,411 & 0,024 \\
\hline
\end{tabular}

*) Correlation analysis uses the Spearman correlation test because the data are not normally distributed

\section{V.DISCUSSION}

The study was conducted at the Department of Clinical Pathology of the USU FK / H. Adam Malik Hospital Medan in collaboration with the Department of Anesthesiology \& Intensive Therapy of the USU FK / H. Adam Malik Hospital Medan. The study was conducted for 3 months from April to July 2019, of 30 patients who had gone through the process of inclusion and exclusion. The number of patients in the study sample were 30 patients ( 19 male and 11 female). Of all study participants, they had a median age of 50 years. The youngest age is 23 years and the oldest is 64 years. PCT levels with a median value of 50.5 , with the lowest level 0 and the highest level 98.34.

It is different from research conducted by Hamed et al 2017. Diagnostic value of Pentraxin-3 in patients with sepsis and septic shock in accordance with the latest sepsis-3 definitions. They conducted a study of 213 patients with sepsis and septic shock. Their study found that of the 73 sepsis patients, 47 $(64 \%)$ of those suffering from sepsis were men and 26 (36\%) were women with an average age of 65 years. Whereas out of 140 septic shock patients 101 (72\%) patients with septic shock 
were male and 39 (28\%) were women with an average age of 67 years. Whereas PCT levels in 73 septic patients with an average value of $12.5 \pm 2.7$, PCT levels in 101 septic shock patients with an average value of $31 \pm 5.7$ and with $p$ values $<0.05$.

There are several risk factors that are considered to play a role in the incidence of sepsis: age, sex, race, comorbid disease, genetics, corticosteroid therapy, chemotherapy, and obesity. At a young age can provide a better inflammatory response than old age. Age is a free risk factor that determines the cure and death rates from this sepsis condition. In the infantpediatric category, the younger the age, the survival rate will be smaller, in contrast to the adult category, the higher the age, the higher the mortality rate.

Sepsis is a systemic response to infection by bacterial microbial agents which will include proinflammatory mediators such as TNF- $\alpha$ and IL-6. Making a faster initial diagnosis of the state of sepsis can reduce mortality and morbidity and the accuracy of antibiotics. Of the various markers of bacterial infection, PCT is a specific marker of severe bacterial infection and can distinguish between sepsis and SIRS (systemic inflammatory response syndromes). Procalcitonin (PCT) is a precursor of the calcitonin hormone found in $\mathrm{C}$ cells of the thyroidal gland and on extratiroidal neurohormone cells. At the beginning of sepsis there will be an increase in PCT concentrations and this increase only occurs in infections caused by bacteria rather than by viruses.

\section{DESCRIPTIVE ANALYSIS OF UREUM, CREATININE, BUN AND NGAL LEVELS DAYS 1 AND 3}

Descriptive analysis shows the Median due to data variables that are not normally distributed, the statistical analysis shows the levels of ureum, creatinine and BUN on day 3 with average values and higher median values when compared with the average and median values on day 1 . Whereas the NGAL level also shows an average value and a median value that is higher when compared to the average value and the median value of the NGAL content on day $l$.

In contrast to research conducted by Fodor et al. 2015. Plasma Neutrophil Gelatinase Associated Lipocalin (NGAL) - Early Biomarkers for Acute Kidney Injury in Critically Ill Patients. They conducted a study of 32 patients with sepsis who were treated in the ICU who were at risk of developing AKI from January to March 2015. NGAL, creatinine and creatinine clearance were examined for all sepsis patients who entered 48 hours and 5 days in ICU and correlated with AKI criteria. Their results show an average value of $1.27 \pm 1.14$ with a minimum value of 0.0 and a maximum value of 4.52 . The measurement of creatinine clearance showed an average value of $0.48 \pm 0.33$ with a minimum value of 0.0 and $a$ maximum value of 1.04 . While the measurement of NGAL levels showed an average value of $3.01 \pm 2.73$ with a minimum value of 0 and a maximum value of 11.91 . The conclusion of Fodor et al's research is that NGAL levels can be a predictive marker for AKI.

In clinical practice at this time still using serum creatinine as a marker of kidney function. But the use of creatinine as an indicator in the early stages of AKI is doubtful, because serum creatinine levels are affected by variations in age, sex, muscle mass, drugs and hydration status, in addition, serum creatinine levels can remain unchanged even though the kidneys have lost more function of $50 \%$. Based on AKIN criteria, the diagnosis of AKI is categorized as a sudden decrease (within 48 hours) of kidney function characterized by an absolute increase in serum creatinine $>0.3 \mathrm{mg} / \mathrm{dL}$, an increase in serum creatinine $>50 \%$ or a decrease in urine output to $<0.5 \mathrm{ml} / \mathrm{kg}$ per hour for $>6$ hours. Serum dilution after volume expansion may be a major factor limiting creatinine's ability to diagnose AKI. ${ }^{10}$

AKI is one of the complications that often arises in patients with critical illness in the intensive care room. AKI due to sepsis is characterized by a progressive decrease in the ability of filtration and elimination of nitrogen metabolism, usually occurring for several hours to several days after the onset of sepsis. In current clinical practice, AKI is usually diagnosed by measuring serum creatinine. Unfortunately, creatinine is a less reliable indicator during acute changes in kidney function. Serum creatinine has many limitations in diagnosing AKI, especially within the scope of the intensive care unit so it is less sensitive to describe the degree of kidney dysfunction in critically ill patients. Early detection of kidney damage in critically ill patients contributes significantly to the management of the patient's management. NGAL has the potential to be an ideal biomarker for early kidney damage detection in critically ill patients. Because NGAL synthesis is significantly induced in the injured epithelium. NGAL concentrations will increase in the serum of patients with acute bacterial infections. NGAL levels increase significantly in AKI patients 24 to 48 hours before the increase in serum creatinine is detected. ${ }^{11,12}$

In line with the results of Hidayat et al (2012) research, the urine NGAL levels of sepsis patients with AKI were significantly higher than those of sepsis without AKI (3,380 ng / $\mathrm{mL}$ compared to $116 \mathrm{ng} / \mathrm{mL} ; \mathrm{p}<0.001$ ). At the cut-off point of $107 \mathrm{ng} / \mathrm{mL}$, urine NGAL has a sensitivity of $100 \%$, specificity of $36 \%$, positive predictive value (PPV) of $60.9 \%$, negative predictive value (NPV) of $100 \%$, and accuracy of $68 \%$. Conclusion, urine NGAL levels have good validity and can be used as a marker of diagnosis of AKI in patients with sepsis. ${ }^{12}$

\section{ANALYSIS OF RELATIONSHIP OF NGAL LEVELS WITH PCT, UREUM, CREATININE AND BUN DAYS 1 AND 3}

Spearman correlation test statistic test shows only NGAL level l day has a significant relationship with PCT level l day, with a value $(r=0.912)$ and $a$ value of $p<0.05$. While the levels of urea, creatinine and BUN do not have a significant relationship. With a value of $\mathrm{p}>0.05$.

In line with research conducted by Wang et al 2014 . Diagnostic and prognostic values of neutrophil gelatinaseassociated lipocalin, matrix metalloproteinase-9, and tissue inhibitors of matrix metalloproteinases-l for sepsis in the Emergency Department: an observational study. They conducted a study of 480 sepsis patients in emergency from February to August 2013. Patients were classified into four groups: SIRS, sepsis, severe sepsis, and septic shock. Serum NGAL, MMP-9, TIMP-1 and PCT were measured, and MEDS scores were calculated at registration. Prognostic values of NGAL, MMP-9 and TIMP-1 are compared with PCT and MEDS scores. Patients were followed up 28 days. Their results showed that NGAL levels were associated with an increase in PCT in SIRS, Sepsis, Severe sepsis and shock septic patients with $p$ values $<0.05^{13}$

NGAL levels have a correlation with PCT because Sepsis is a systemic response to infection by bacterial microbial agents that will include proinflammatory mediators such as TNFIFN and IL-1 and IL-6. Where this complex process begins when gram-negative bacterial endotoxins enter and infect tissues so that severe inflammatory processes occur that are systemic, and the degree of severity of inflammation also occurs due to dysregulation of the host immune system to the infection response. Tissue damage that arises will activate the coagulation system, so that microtrombus will occur in microcirculation, which will eventually disrupt the perfusion of organs including the kidneys. As a result of decreased perfusion in the kidneys, there will be injury to glomerular epithelial cells and tubules, this process is also influenced by 
cytokines, growth factors, neutrophil movement, macro phages, eosinophils to the injured area. When neutrophy infiltration in an area of inflammation or injury, the neutrophyl will release NGAL in response to tissue damage. ${ }^{14}$

Simultaneously when there is an increase in systemic inflammatory activity involving pro-inflammatory cytokines such as TNF- $\alpha$, IFN $\alpha$ and IL-1 and IL-6. Then this will be responded by thyroid gland $\mathrm{C}$ cells and extratiroidal neuroh ormone cells to produce PCT as a systemic inflammatory response in sepsis. And because sepsis is generally caused by gram-negative bacteria, an increase in PCT concentration only occurs in infections caused by bacteria rather than by viruses. In normal conditions without infection, PCT is only present in C cells in the thyroid gland, but when there is bacterial infection an increase in PCT concentration will occur. This happens because if there is no infection transcription CALC-I genes from extratiroidal will be suppressed. When bacterial infection occurs, CALC-I gene genes from CT-RNA messager will be expressed from various extratiroidal neuroendokrine cells (parenchymal tissue) in the body so that all PCT molecules will spread systemically. ${ }^{14}$

While the measurement of NGAL day 3 had a significant relationship with PCT day 3 , with a value $(r=0.0771)$ and $\alpha p$ value $<0.001$. Likewise, measurement of NGAL day 3 has a significant relationship with levels of ureum, creatinine and BUN day 3 , with each $p$ value $<0.05$. So it can be concluded NGAL level 3 has a positive correlation and significant correlation with PCT, urea, creatinine and BUN levels.

In line with research conducted by Corbacioglu et al 2017. The value of plasma neutrophil gelatinase-associated lipocalin (NGAL) in distinguishing between acute kidney injury (AKI) and chronic kidney disease (CKD). They conducted prospective studies with the aim of using NGAL levels as biomarkers to differentiate AKI and CKD. The study was conducted on 25 AKI patients, 22 patients with stable CKD and 22 control populations. All BUN, creatinine, plasma NGAL levels were examined. Their results showed that higher NGAL levels were found in AKI patients compared with stable CKD patients, with a palue $<0.05$. NGAL levels can be as biomarkers to differentiate AKI from CKD with a cut off of 457 $\mathrm{ng} / \mathrm{ml}^{15}$

My results are also in line with research conducted by Cuertero et al 2018. Does Whole-Blood Neutrophil GelatinaseAssociated Lipocalin Stratify Acute Kidney Injury in Critically Ill Patients?. The study was conducted prospectively, with the aim of assessing NGAL levels as AKI biomarkers in sepsis and without sepsis patients. Plasma NGAL levels were examined 48 hours after the patient entered ICU treatment. Patients were classified according to AKIN and KDIGO. Their results showed 55 patients with NGAL levels $<178 \mathrm{ng} / \mathrm{ml}$ (negative test), while 45 patients with NGAL levels $>178 \mathrm{ng} / \mathrm{ml}$ (positive test). There was a significant correlation between creatinine clearance between the two populations, where creatinine clearance was lower in patients with NGAL levels $>178 \mathrm{ng} / \mathrm{ml}$ with p values $<0.05$. $^{16}$

The biological marker of AKI can be a component of serum or urine. Urine biological markers are promising to detect early AKI, so it can be estimated early, therefore, it can be useful for early diagnosis, identification of mechanism disorders, and determination of the location and severity of dysfunction. The term biological marker was first explained in 1989, which means measurable indicators for certain biological conditions and for certain disease processes. In addition, the US Food and Drug Administration (FDA) uses the term biological marker to describe each assess risk or disease. An ideal biological marker for AKI must be affordable, fast and easy to measure, precise and accurate, and can determine the severity of dysfunction, specifically for the kidneys, increasing in the early stages of dysfunction, with high sensitivity and specificity. Of the many new biological markers available, there are biological markers that are currently widely used by clinicians around the world to detect AKI, including Neutrophil GelatinaseAssociated Lipocalin (NGAL) . ${ }^{17}$

NGAL is a $25 \mathrm{kD}$ molecular weight protein bound to gelatinase from neutrophil cells. In normal circumstances NGAL is excreted in very low levels from various body tissues, such as kidneys, lungs, stomach and colon. NGAL is the most rapid type of protein and is significantly increased due to interference (injury) in the kidneys or the occurrence of nephrotoxic processes. The process of injury to the kidneys will stimulate inflammation and release of cytokines such as TNF $\alpha$, IFN $\alpha$, IL-1.IL-2.IL-6, release of growth factors due to interactions with the innate good immune system, in which the role of moving neutrophyl and infiltration the area of inflammation that releases its toxic granules and at the same time releases NGAL which this process will cause endothelial damage, free radical scavenging and cellular immunity system that is directed against $\mathrm{T}$ cells, at the same time the inflammatory process will also stimulate the coagulation system resulting in microtrombus in the kidney circulation. As a result of this process there will be disruption of blood flow to the kidneys, so that it will disrupt the kidneys functionally, in this case it can be seen an increase in urea and creatinine as one form of functional disorders. NGAL can be examined from blood or urine and can be used to establish an early diagnosis of AKI because it can be detected 1-2 days before the increase in serum creatinine. NGAL does not significantly increase in Chronic Kidney Disease (CKD).$^{18}$

In my research, there is a significant relationship between NGAL and sepsis (enforcement of sepsis using PCT). From the statistical analysis, the NGAL level tends to increase on day 3 in sepsis patients treated in the ICU, so that these patients have complications for the occurrence of AKI. Acute kidney injury (AKI) is a frequent and severe complication in septic patients in the ICU. Moreover, there is strong evidence between sepsis and sepsis shock as a cause of AKI in critically ill patients. About $50 \%$ or more patients in the ICU will develop AKI with a high mortality rate. The Beginning and Ending Supportive Therapy (BEST) study, which evaluated 54 hospitals in 23 countries, found evidence that sepsis was the most common cause of AKI in critically ill patients (47.5\%) and reported that $13.8 \%$ of patients AKI patients who undergo hemodialysis (HD) while being treated, after being discharged from the hospital, apparently must continue hemodialysis. $^{19}$

Sepsis and sepsis shock are important risk factors for AKI, especially in patients with critical illness, often ending in death despite resuscitation or renal replacement therapy. The combination of sepsis and acute kidney disorders becomes a serious problem, so it becomes a substance that needs to be understood where sepsis is closely related to the occurrence of acute kidney disorders, which affects the length of hospital stay, the magnitude of treatment and the risk of death. ${ }^{19}$

Increased NGAL in sepsis patients is closely related to the definition and pathophysiology of complex sepsis, the process begins when the interaction between bacterial products in the form of toxins (both endotoxins and exotoxins as super antigens), viruses, parasites, tissue damage (external factors) with host factors called host immune response includes humoral and cellular defense factors. ${ }^{19}$ 
gram-negative endotoxins, or gram-positive exotoxins. Endot oxins are Lipopolysaccharides (LPS) derived from gramnegative bacterial cell walls are very powerful inflammatory cascade stimulators and are the triggers for sepsis. Gramnegative bacteria produce endotoxins chemically called lipopolysaccharides, along with patient antibodies to form LPSab (Lipopolisakaride Antibody). LPSab in the blood by the mediation of CD14 receptors activates macrophages via NF $\beta$. Then macrophages will express proinflammatory cytokines IL- 1, TNF- $\alpha$, and IL- 6 which cause inflammatory reactions. The above reactions occur mostly in gram negative bacteria that have LPS on their walls. ${ }^{19}$

Sepsis stimulates Nitric Oxide Syntase (NOS) to Nitric Oxide (NO) which causes arterial dilatation. Arterial vasodilation causes a decrease in vascular resistance, increased sympathetic tone and release of vasopressin from the central nervous system through activation of the Renin Angiotensin Aldosteron system (RAA), which induces water retention and predisposes to the onset of acute renal impairment. Sepsis also induces radical oxygen formation which causes nitric oxyde in the kidney endothelium to become peroxy nitrite, resulting in necrosis and acute tubular injury. In the proinflammatory phase such as cytokines and chemokines cause further acute damage to the kidney endothelium thereby increasing mortality. ${ }^{20}$

\section{CONCLUSION}

There was a significant relationship between NGAL on day 1 and PCT on day 1 . There was a significant relationship on NGAL on day 3 with PCT on day 3, there was a significant relationship on NGAL on day 3 with urea, creatinine and BUN on day 3.

\section{REFERENCE}

1. Dellinger RP, Levy MM, Rhodes A, Annane D, Gerlach H, Opal SM, et al. Surviving sepsis campaign: international guidelines for management of severe sepsis and septic shock: 2012. Crit Care Med. 2013; 41:580-637.

2. Fleischmann C, Scherag A, Adhikari NK, et al. International Forum of Acute Care Trialists. Assessment of global incidence and mortality of hospitaltreated sepsis: current estimates and limitations. Am J Respir Crit Care Med. 2015

3. de Geus HR, Betjes MG, Schaick R and Groeneveld JA: Plasma NGAL similarly predicts acute kidney injury in sepsis and nonsepsis. Biomarkers Med 7: 415-421, 2013

4. Devarajan P. Neutrophil gelatinase-associated lipocalin (NGAL): a new marker of kidney disease. Scand J Clin Lab Invest 2015;68:89-94.

5. Bellomo R, Ronco C, Mehta RL, Asfar P. Acute kidney injury in the ICU: from injury to recovery: reports from the 5th Paris International Conference. Bellomo et al. Ann. Intensive Care (2017) 7:49

6. Hamed-Fielitz A, Bellomo R, Devarajan P et al. The predictive performance of plasma neutrophil gelatinase-associated lipocalin (NGAL) increases with grade of acute kidney injury. Nephrol Dial Transplant 2015;24(11):3349-3354

7. Kane-Gill SL, Sileanu FE, Murugan R, Trietley GS, Handler SM, Kellum JA, et al. Risk factors for acute kidney injury in older adults with critical illness: a retrospective cohort study. Am J Kidney Dis. 2015; 65:860-9.

8. Lopes J, Jorge S, Resina C, Santos C, Pereira I, Neves J, Antunes F, et al. Acute kidney injury in patients with sepsis: a contemporary analysis. International Journal of Infectious Diseases. 2016;13: 176-181

9. Fodor C, Scherag A, Adhikari NK, et al. International Forum of Acute Care Trialists. Assessment of global incidence and mortality of hospital-treated sepsis: current estimates and limitations. Am J Respir Crit Care Med. 2015

10. Ghonemy TA and Amro GM: Plasma neutrophil gelatinase-associated lipocalin (NGAL) and plasma cystatin C (CysC) as biomarker of acute kidney injury after cardiac surgery. Saudi J Kidney Dis Transpl 25: 582-588, 2014.

11. Kane-Gill SL, Sileanu FE, Murugan R, Trietley GS, Handler SM, Kellum JA, et al. Risk factors for acute kidney injury in older adults with critical illness: $\alpha$ retrospective cohort study. Am J Kidney Dis. 2015; 65:860-9.

12. Lopes J, Jorge S, Resina C, Santos C, Pereira I, Neves J, Antunes F, et al. Acute kidney injury in patients with sepsis: a contemporary analysis. International Journal of Infectious Diseases. 2016;13: 176-181

13. Lagos-Arevalo P, Palijan A, Vertullo L, Devarajan P, Bennett MR, Sabbisetti V, Bonventre JV, Ma Q, Gottesman RD and Zappitelli M: Cystatin C in acute kidney injury diagnosis: Early biomarker or alternative to serum creatinine? Pediatr Nephrol 30: 665-676, 2015

14. Lameire N, Biesen WV, Vanholder R. The rise of prevalence and the fall of mortality of patients with acute renal failure: what the analysis of two databases does and does not tell us. J Am Soc Nephrol. 2016;17:923-5.

15. Marshall JC, Naqbi AL. Principles of source control in the management of sepsis. Crit Care Nurs Clin North Am. 2014 Mar;23(1):99-114.

16. Mandelbaum T, Scott DJ, Lee J, Mark RG, Malhotra A, Waikar SS, et al. Outcome of critically ill patients with acute kidney injury using the Acute Kidney Injury Network criteria. Crit Care Med. 2014; 39:2659-64.

17. Mishra J, Mori $\mathrm{K}, \mathrm{Ma} \mathrm{Q}$, et al. Neutrophil gelatinase-associated lipocalin
(NGAL): a novel urinary biomarker for cisplatin nephrotoxicity. Am J Nephrol 2004;24:307-315

18. Merik EP, Taeb MA. qSOFA and new sepsis definition. J Thorac Dis. 2017 Apr; 9(4): 943-945

19. Parikh AG, Moledina DG, Coca SG, Philbrook TS and Garg AX. Application of new acute kidney injury biomarkers in human randomized controlled trials. Kidney International. 2016 ;89: 1372-1379

20. Rennie TJW, De Souza N, Donnan PT, Marwick CA, Davey P, Dreischulte T, et al. Risk of acute kidney injury following community prescription of antibiotics: self-controlled case series. Nephrol Dial Transplant. 2018 Jun 28 